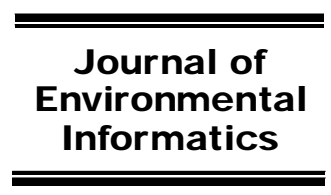

www.iseis.org/jei

\title{
Interval Binary Programming for Noise Control within an Urban Environment
}

\author{
W. Huang ${ }^{1, *}$, L. M. Dai ${ }^{2}$, B.W. Baetz ${ }^{1}$, M. F. Cao ${ }^{2}$, and S. Razavi ${ }^{1}$ \\ ${ }^{1}$ Department of Civil Engineering, McMaster University, Hamilton, ON L8P 2R3, Canada \\ ${ }^{2}$ Faculty of Engineering and Applied Science, University of Regina, Regina, Saskathewan S4S 0A2, Canada
}

Received 12 April 2012; revised 10 July 2012; accepted 12 September 2012; published online 27 June 2013

\begin{abstract}
This paper introduces an interval binary programming (IBP) method to the selection of control measures for noise reduction under uncertainty, by incorporating the concepts of interval numbers and interval mathematical programming into a binary programming optimization framework. As an extension of the binary programming method, IBP can explicitly address complexities and uncertainties in a noise control system. Parameters in the IBP model can be expressed as intervals, and uncertainties are effectively incorporated within the model solution process. The modelling approach is applied to a representative control measure selection problem for noise reduction in an urban environment. Results of the application indicate that useful solutions for noise control practices can be generated. A number of decision alternatives have been obtained and analyzed under different acceptable noise levels for two communities, and they reflect complex tradeoffs between environmental and economic considerations.
\end{abstract}

Keywords: interval binary programming, uncertainty Analysis, noise control, system optimization

\section{Introduction}

The problems associated with acoustic noise continues to be a major challenge for urban communities throughout the world due industrialisation and urbanisation. The noise generated from plants and factories can pose significant threats to the health of workers and residents in nearby communities (King and Davis, 2003). Noise-induced hearing loss is one of the most common occupational diseases and the second most self-reported occupational illness or injury in the United States (Murray-Johnson et al., 2004). According to the National Institute for Occupational Safety and Health (NIOSH), approximately 30 million U.S. workers are currently exposed to noise hazards on the job and an additional 9 million U.S. workers are at-risk for developing hearing loss (NIOSH, 1998). Long-term exposure to excessive noise levels is recognized as the major cause of hearing loss. Since hearing loss is difficult to cure, appropriate engineering controls are strongly recommended to minimize noise and diminish the noise effect on workers and nearby residences. However, engineering controls differ in cost and noise reduction capability; more effective noise control measures usually require greater investment, while less effective measures may have lower costs. Therefore, optimization models are desired for helping decision makers make tradeoffs between system cost and noise control efficiency.

\footnotetext{
* Corresponding author. Tel.: +1 306 5913508; fax: +1 9055299688. E-mail address: wendywanzhihuang@gmail.com (W. Huang).
}

ISSN: 1726-2135 print/1684-8799 online

(C) 2013 ISEIS All rights reserved. doi:10.3808/jei.201300236
In the past decades, significant efforts have been made in developing optimization models for noise control systems. For example, Yeh et al. (2004) developed an optimization model for noise reduction in a multiple noise system by using a genetic algorithm. Asawarungsaengkul and Nanthavanij (2006) proposed six optimization models for identifying the optimal noise hazard control strategy, including two models for engineering controls, two for job rotation and two for the use of hearing protection devices. They then applied an algorithmic approach to the selection of engineering controls for optimal noise redution (Asawarungsaengkul and Nanthavanij, 2007). Zachary et al. (2010) developed a multi-impact optimization model to reduce aviation noise and emissions at Luxembourg's Findel Airport. Prats et al. (2011) proposed a multiobjective optimization model for designing aircraft noise abatement strategies. Also, there are a number of other optimization models for identifying optimal noise control strategies (Waly and Sarker, 1998; King and Davis, 2003; Mun and Cho, 2009; Tokmechi, 2011).

In a practical noise control system, many parameters such as noise-reduction effects of different control measures, the unit cost of each measure, and acceptable noise levels for receptors may have some levels of uncertainty. However, previous optimization models are deterministic and only deal with parameters with crisp values. Therefore, in this paper, an interval binary programming method will be developed and applied to a representative noise control system for selecting optimal noise reduction measures. Interval solutions for binary variables will be analyzed and interpreted to provide useful decision alternatives for controlling noise from different sources and thus demonstrate the potential applicability of the developed method. 


\section{Methodology}

\subsection{Interval Linear Programming (ILP)}

In ILP, interval values are allowed to be communicated into the optimization process. All parameters and decision variables in a linear programming model can be intervals (Huang et al., 1992).

Specifically, an ILP model can be defined as follows:

$\operatorname{Min} / \operatorname{Max} f^{ \pm}=\sum_{j=1}^{n} c_{j}^{ \pm} x_{j}^{ \pm}$

Subject to:

$\sum_{j=1}^{n} a_{i j}^{ \pm} x_{j}^{ \pm} \leq b_{i}^{ \pm}, i=1,2, \ldots, m$

$x_{j}^{ \pm} \geq 0, j=1,2, \ldots, n$

where $a_{i j}^{ \pm}, b_{i}^{ \pm}$, and $c_{j}^{ \pm} \in R^{ \pm}$( $R^{ \pm}$denotes a set of intervals). Definitions of interval, the related properties, and operation principals are shown in Appendix 1.

According to Huang et al. (1992, 1995), an interactive solution algorithm named the two-step-method (TSM) was proposed to solve the above problem. Interval solutions can be obtained based on the analysis of detailed interrelationships between the parameters and variables and between the objective function and constraints. The main idea of the TSM is to convert the original ILP model into two LP submodels corresponding to the lower and upper bounds of the objecttive-function value, respectively. In detail, when the objective function of model (1) is to be maximized, the first submodel would correspond to the upper bound of equation (1a). It can be formulated as follows (assume that $b_{i}^{ \pm}>0$ and $f^{ \pm}>0$ ):

$\operatorname{Max} f^{+}=\sum_{j=1}^{k} c_{j}^{+} x_{j}^{+}+\sum_{j=k+1}^{n} c_{j}^{+} x_{j}^{-}$

Subject to:

$\sum_{j=1}^{k}\left|a_{i j}^{ \pm}\right|^{-} \operatorname{Sign}\left(a_{i j}^{ \pm}\right) x_{j}^{+}+\sum_{j=k+1}^{n}\left|a_{i j}^{ \pm}\right|^{+} \operatorname{Sign}\left(a_{i j}^{ \pm}\right) x_{j}^{-} \leq b_{i}^{+}, i=1,2, \ldots, m$

$x_{j}^{+} \geq 0, j=1,2, \ldots, k$

$x_{j}^{-} \geq 0, j=k+1, k+2, \ldots, n$

where for $n$ interval coefficients $c_{j}^{ \pm}(j=1,2, \ldots, n)$ in the objective function of the model, we assume the former $k$ coefficients of them are positive, and the latter $(n-k)$ coefficients are negative, i.e. $c_{j}^{ \pm} \leq 0 \quad(j=k+1, k+2, \ldots, n)$. Solutions of $x_{\text {jopt }}^{+}(j=1,2, \ldots, k)$ and $x_{\text {jopt }}^{-}(j=k+1, k+2, \ldots$, n) can be obtained through solving sub-model (2). Based on solution for submodel (2), the submodel corresponding to the lower bound of equation (1a) can be formulated as follows:

$\operatorname{Max} f^{-}=\sum_{j=1}^{k} c_{j}^{-} x_{j}^{-}+\sum_{j=k+1}^{n} c_{j}^{-} x_{j}^{+}$,

subject to:

$\sum_{j=1}^{k}\left|a_{i j}^{ \pm}\right|^{+} \operatorname{Sign}\left(a_{i j}^{ \pm}\right) x_{j}^{-}+\sum_{j=k+1}^{n}\left|a_{i j}^{ \pm}\right|^{-} \operatorname{Sign}\left(a_{i j}^{ \pm}\right) x_{j}^{+} \leq b_{i}^{-}, i=1,2, \ldots$,

$m$

$0 \leq x_{j}^{-} \leq x_{j \text { opt }}^{+}, j=1,2, \ldots, k$

$x_{j}^{+} \geq x_{j \text { opt }}^{-}, j=k+1, k+2, \ldots, n$

From submodel (3), Solutions of $x_{\text {jopt }}^{-}(j=1,2, \ldots, k)$ and $x_{\text {jopt }}^{+}(j=k+1, k+2, \ldots, n)$ can be obtained. Thus, the final solution of $f_{\text {opt }}^{ \pm}=\left[f_{\text {opt }}^{-}, f_{\text {opt }}^{+}\right]$and $x_{\text {jopt }}^{ \pm}=\left[x_{\text {jopt }}^{-}, x_{\text {jopt }}^{+}\right]$can be obtained for model (1).

\subsection{Interval Binary Programming}

In integer programming, all the decision variables are integers. An integer programming model can be formulated as follows:

$\operatorname{Min} / \operatorname{Max} f=\sum_{j=1}^{n} c_{j} y_{j}$

Subject to:

$\sum_{j=1}^{n} a_{i j} y_{j} \leq b_{i}, i=1,2, \ldots, m$

$y_{j} \geq 0$ and $y_{j}=$ integer variable, $\mathrm{j}=1,2, \ldots, n$

where $a_{i j}, b_{j}$ and $c_{j} \in \mathrm{R}$.

A well-known approach to solve integer programming problems is the branch-and-bound algorithm (Garfinkel and Nemhauser, 1972; Blair and Jeroslow, 1982; Achterberg, 2007). Mixed integer linear programming (MILP) has been widely used in many engineering fields since it can deal with capacity-expansion issues (Baetz, 1990; Rajagopalan et al., 1998; Chen et al., 2002). However, it may not be effective when uncertain parameters exist (Jenkins, 1982). Therefore, Huang et al. (1995) introduced an interval mixed integer linear programming (IMILP) method, where input uncertainties can be expressed as integer-intervals and/or intervals. In the IMILP model, the integer-intervals are interval binary variables, indicating capacity-expansion options. Therefore, as an extension of the IMILP model, an interval binary programming (IBP) method can be formulated as follows: 
$\operatorname{Min} / \operatorname{Max} f^{ \pm}=\sum_{j=1}^{n} c_{j}^{ \pm} x_{j}^{ \pm}$

Subject to:

$\sum_{j=1}^{n} a_{i j}^{ \pm} x_{j}^{ \pm} \leq b_{i}^{ \pm}, i=1,2, \ldots, m$

$x_{j}^{ \pm} \geq 0, j=1,2, \ldots, n$

$x_{j}^{ \pm}=0$ or $1, j=1,2, \ldots, n$

The solutions of the interval binary varibles have four possible presentations $([0,0],[1,1],[0,1]$, and $[1,0])$. They can represent the related interval decisions (for optimal scenarios) that can be interpreted to provide decision alternatives reflecting system condition variation caused by input uncertainties (Huang et al., 1995).

These methodologies can be used to optimize the cost and effectiveness in the selection of noise-reducing technologies to mitigate the noise pollution problem outlined above. Given costs and the noise reduction potentials of each type of technology, IBP can be used to select the optimal combination of technologies to most efficiently achieve a desired level of noise reduction.

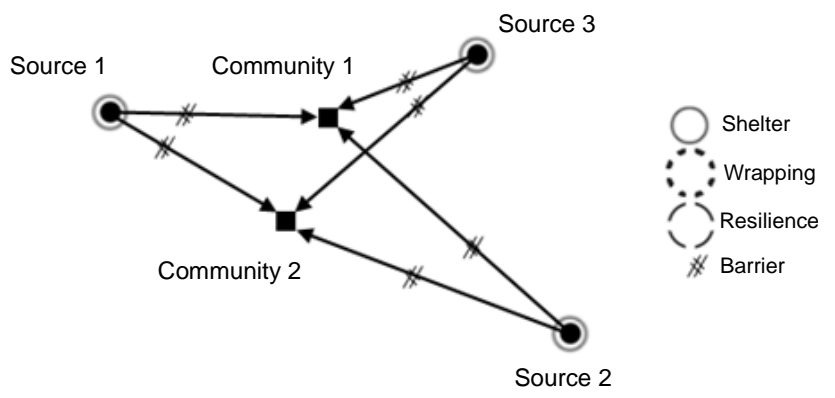

Figure 1. The study system.

\section{Application}

\subsection{Overview of the system}

A representative problem has been developed to illustrate the IBP modelling approach, based upon cost and technical data extracted from the noise control system literature. Noise sources considered in the studied system are factories and the receivers are adjoining communities. Amounts of sources and receivers are defined as $I$ and $K$, respectively. The original generated noise levels in the factories and the acceptable noise levels in the communities are shown as $O N_{i}(\mathrm{~B})$ and $A N_{k}(\mathrm{~B})$, respectively. The distance between factory $i$ and community $k$ is denoted as $\mathrm{D}_{i k}(\mathrm{~km})$, and a barrier could be established to mitigate noise. Figure 1 presents the hypothetical noise control system. Three noise sources and two affected communities are considered in this application. There are several alternatives that could be used to control noise from the original sources. In this case, four external noise control measures, as well as equipment updates are considered as the potential options for controlling noise, as presented in Table 1. These four external control measures can also be combined with each other to enhance the effect of noise-reduction.

Table 1. Noise Control Measures for Factory $i$

\begin{tabular}{llll}
\hline $\begin{array}{l}\text { Option } \\
(\mathrm{j})\end{array}$ & $\begin{array}{l}\text { Noise control } \\
\text { measures }\end{array}$ & $\begin{array}{l}\text { Noise-reduction } \\
\text { effect } \mathrm{RE}_{\mathrm{j}}(\mathrm{dB})\end{array}$ & Cost \\
\hline 1 & Shelter & {$[9,10]$} & {$[190,210]$} \\
2 & Wrapping & {$[7.5,8]$} & {$[100,110]$} \\
3 & Resilience & {$[5.6,6]$} & {$[55,60]$} \\
4 & Barrier & {$[11,12]$} & {$[240,260]$} \\
5 & Equipment update & {$[23,25]$} & {$[600,650]$} \\
6 & Shelter + resilience & {$[13.8,14.5]$} & {$[260,280]$} \\
7 & Shelter + wrapping & {$[15,16]$} & {$[320,350]$} \\
8 & Shelter + Barrier & {$[20.5,22]$} & {$[520,550]$} \\
9 & Wrapping + Resilience & {$[10,11]$} & {$[200,220]$} \\
10 & Wrapping + Barrier & {$[18,20]$} & {$[400,435]$} \\
11 & Resilience + Barrier & {$[16,18]$} & {$[350,370]$} \\
\hline
\end{tabular}

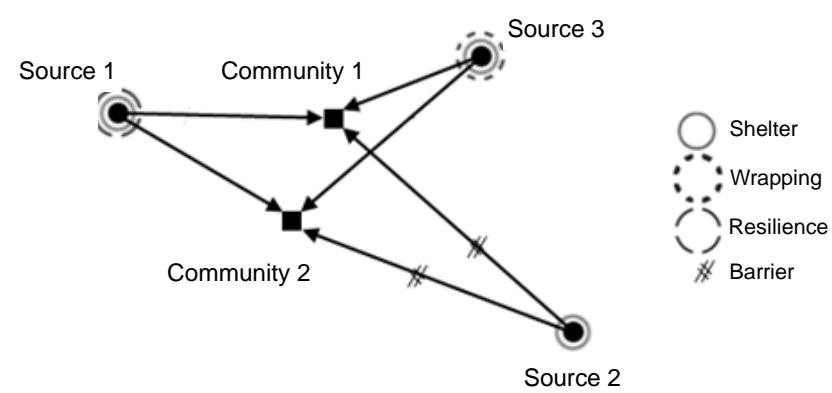

Figure 2. Noise control measures under Scenario 1.

Eleven noise control options could be generated, as shown in Table 1. The unit cost for each combination of noise control is denoted as $R E_{j}(\mathrm{~B})$. As for the noise-reduction effect, it is related to the noise sources and noise control measures, thus $C_{i j}(\$ / \mathrm{B})$ is employed to represent this effect.

Table 1 shows the potential noise control measures and their combinations, and their related noise-reduction effect and unit cost for each measure. In this study, eleven control measures are considered for reducing excessive noise from different sources. These control measures include sheltering, wrapping, resilience features, barriers and combinations of the above methods. Equipment update is also one potential measure to control noise. The sheltering method reduces emitted noise by placing the noise sources inside a shelter; for example, housing noise-generating pieces of equipment inside a building. Wrapping is similar to sheltering, but instead of placing the sources inside a shelter, each source is separated from the environment by wrapping a noise absorbing material around it. Resilience features are noise control measures that reduce noise generated by placing padding and noise reducing materials, thus reducing noise generated from moving parts in the equipment. Barriers separate the source and the receiver 
through placement of a physical obstruction between them. The obstruction can absorb or redirect sound pressure, effectively reducing the amount of noise that is heard by the receiver.

Table 2. Acceptable Noise Levels of Considered Communities

\begin{tabular}{lllllll}
\hline Community & $\begin{array}{l}\text { Strict } \\
(\mathrm{dB})\end{array}$ & level & $\begin{array}{l}\text { Normal } \\
(\mathrm{dB})\end{array}$ & level & $\begin{array}{l}\text { Loose } \\
(\mathrm{dB})\end{array}$ & level \\
\hline $1(\mathrm{k}=1)$ & 60 & 61 & 62 & \\
$2(\mathrm{k}=2)$ & 55 & 56 & 57 & \\
\hline
\end{tabular}

Table 3. Distance from the Noise Source i to Community k

\begin{tabular}{llll}
\hline $\begin{array}{l}\text { Distance } \\
(\mathrm{km})\end{array}$ & $\begin{array}{l}\text { Noise source 1 } \\
(\mathrm{i}=1)\end{array}$ & $\begin{array}{l}\text { Noise source 2 } \\
(\mathrm{i}=2)\end{array}$ & $\begin{array}{l}\text { Noise source 3 } \\
(\mathrm{i}=3)\end{array}$ \\
\hline $\mathrm{k}=1$ & 150 & 200 & 120 \\
$\mathrm{k}=2$ & 140 & 180 & 170 \\
\hline
\end{tabular}

Different control measures produce different noise-reduction effects and also have different costs associated to them. The original noise levels of noise sources 1,2 and 3 are [90, $92],[95,97]$ and $[100,102] \mathrm{dB}$, respectively. For the 2 communities considered here, the acceptable noise levels are [60, $62]$ and $[55,57] \mathrm{dB}$, respectively. For each community, 3 scenarios are considered, as shown in Table 2. Table 3 presents the distance from Noise Source $i$ to Community $k$.

\subsection{Interval Binary Programming (IBP) Model for Noise Control System}

The problem under consideration is that excessive noise at emission sources would be reduced by different control measures over a given planning horizon. Binary variables $\left(\mathrm{X}_{i j}\right)$ are introduced to denote whether or not the noise-control measure is selected. If $X_{i j}$ is equal to one, the $j^{\text {th }}$ noise control option would be implemented for factory $i$. Conversely, if the value of $X_{i j}$ is zero, the corresponding options would not be implemented. In the case of interval binary solutions, $[0,1]$ indicates a conservative model and $[1,0]$ indicates an optimistic model. In addition, for factory $i$, no more than one of the $J$ options can be implemented. Since this problem has several configurations of options - acceptable noise levels, combinations of mitigation methods - this paper will use a series of scenarios to describe sets of conditions. There are three noise scenarios: strict, normal, and relaxed. The objecttive is to minimize the total noise-reduction cost (TC) for identifying effective noise-control measures while the noise received in the considered communities is no higher than acceptable levels. Thus, the following optimization model can be formulated:

$\operatorname{Min} \mathrm{TC}=\sum_{i=1}^{I} \sum_{j=1}^{J} C_{i j} X_{i j}$

Subject to:

(1) The noise received in community $k$ should be no higher than the accepted level $\left(A N_{k}\right)$. Noise reduction in the atmosphere is affected by a number of factors, including temperature and air pressure. At standard atmospheric conditions, noise reduction is $158.8 \mathrm{~dB}$ per kilometer, giving us:

$\lg \left\{\sum_{i=1}^{I} 10^{\left[\left(O N_{i}-\frac{158.8 D_{i k}}{1000}-\sum_{j=1}^{J} X_{i j} R E_{j}\right) / 10\right]}\right\} \leq A N_{k}, k=1,2, \ldots, K$

Noise levels are indicated by decibels, which is on a logarithmic scale. The source noise $\left(O N_{i}\right)$ is reduced by distance and the selected noise control measure $\left(X_{i j} R E_{j}\right)$.

(2) The longer the distance, the less the noise effect: For each community, we sort the distances between all the noise sources $(i=1,2, \ldots, I)$ to community $k$ in a descending order, which are denoted as $D_{(i) k}(i=1,2, \ldots, i 1, i 2, \ldots, I)$, such that $D_{(i 1) k} \geq D_{(i 2) k}$. Thus, we have:

$O N_{(i 1)}-\frac{158.8 D_{(i 1) k}}{1000}-\sum_{j=1}^{J} X_{(i 1) j k} R E_{j} \leq O N_{(i 2)}-\frac{158.8 D_{(i 2) k}}{1000}-$
$\sum_{j=1}^{J} X_{(i 2) j k} R E_{j}, \quad(i 1),(i 2)=1,2, \ldots, I ; k=1,2, \ldots, K \quad(6 \mathrm{c})$

(3) No more than one of the $J$ combinations of noise control can be implemented for the combination of factory $i$ and community $k$ :

$\sum_{j=1}^{J} X_{i j} \leq 1, i=1,2, \ldots, I$

(4) Technical constraints:

$X_{i j}=0$ or $1, i=1,2, \ldots, I ; j=1,2, \ldots, J$.

A few assumptions are made in this model in terms of noise transmission. Sound pressure decreases over distance, but this decrease is dependent on air pressure, temperature and humidity. Standard atmospheric conditions are assumed. Secondly, sound waves can be absorbed and deflected by obstacles between the transmitter and receiver. For the purposes of this model, obstacles are ignored and it is assumed that noise travels unhindered between the sources and teh communities. In practical problems, many system parameters related to noise control systems such as unit costs, noise-reduction effects of different control measures, and noise levels from different sources may not be determined as crisp values. Most of them may present some levels of uncertainty. Moreover, the quality of information that can be obtained for these uncertainties is generally not good enough to be presented as probability information. For example, the original noise levels for source 1 may vary with $[90,92] \mathrm{dB}$, which means that the 
lowest level of the original noise from source 1 would be 90 $\mathrm{dB}$ and the highest level would be $92 \mathrm{~dB}$. Based on these considerations, interval parameters are introduced into the noise control optimization model framework to communicate uncertainties in $C_{i j}, O N_{i}$, and $R E_{j}$ into the optimization process. This leads to an interval binary noise control optimization model as follows:

$\operatorname{Min} \mathrm{TC}^{ \pm}=\sum_{i=1}^{I} \sum_{j=1}^{J} C_{i j}^{ \pm} X_{i j}^{ \pm}$

Subject to:

$\lg \left\{\sum_{i=1}^{I} 10^{\left[\left(O N_{i}^{ \pm}-\frac{158.8 D_{i k}}{1000}-\sum_{j=1}^{J} X_{i j}^{ \pm} R E_{j}^{ \pm}\right) / 10\right]}\right\} \leq A N_{k}^{(p)}, k=1,2, \ldots, K$

$O N_{(i 1)}^{ \pm}-\frac{158.8 D_{(i 1) k}}{1000}-\sum_{j=1}^{J} X_{(i 1) j}^{ \pm} R E_{j}^{ \pm} \leq O N_{(i 2)}^{ \pm}-\frac{158.8 D_{(i 2) k}}{1000}-$

$\sum_{j=1}^{J} X_{(i 2) j}^{ \pm} R E_{j}^{ \pm},(i 1),(i 2)=1,2, \ldots, I ; k=1,2, \ldots, K$

$\sum_{j=1}^{J} X_{i j}^{ \pm} \leq 1, i=1,2, \ldots, I$

$X_{i j}^{ \pm}=[0,1],[0,0],[1,0]$ or $[1,1] ; i=1,2, \ldots, I$, and $j=1$, $2, \ldots, J$

According to Huang et al. (1995), model (7) can be transformed to the following two submodels:

Submodel 1:

$\operatorname{Min} \mathrm{TC}^{-}=\sum_{i=1}^{I} \sum_{j=1}^{J} C_{i j}^{-} X_{i j}^{-}$

Subject to

$\lg \left\{\sum_{i=1}^{I} 10^{\left[\left(O N_{i}^{-}-\frac{158.8 D_{i k}}{1000}-\sum_{j=1}^{J} X_{i j}^{-} R E_{j}^{+}\right) / 10\right]}\right\} \leq A N_{k}^{(p)}, k=1,2, \ldots, K$

$O N_{(i 1)}^{-}-\frac{158.8 D_{(i 1) k}}{1000}-\sum_{j=1}^{J} X_{(i 1) j}^{-} R E_{j}^{+} \leq O N_{(i 2)}^{-}-\frac{158.8 D_{(i 2) k}}{1000}-$

$\sum_{j=1}^{J} X_{(i 2) j}^{-} R E_{j}^{+},(i 1),(i 2)=1,2, \ldots, I ; k=1,2, \ldots, K$

$$
\begin{aligned}
& \sum_{j=1}^{J} X_{i j}^{-} \leq 1, i=1,2, \ldots, I . \\
& X_{i j}^{-}=0 \text { or } 1 ; i=1,2, \ldots, I, \text { and } j=1,2, \ldots, J .
\end{aligned}
$$

Submodel 2:

$\operatorname{Min} \mathrm{TC}^{+}=\sum_{i=1}^{I} \sum_{j=1}^{J} C_{i j}^{+} X_{i j}^{+}$

Subject to

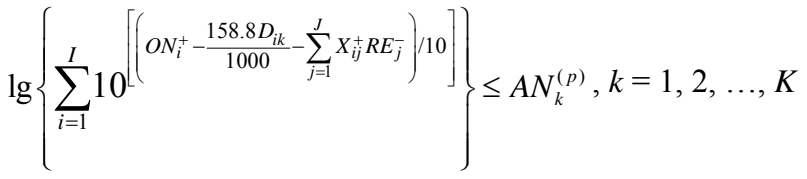

$O N_{(i 1)}^{+}-\frac{158.8 D_{(i 1) k}}{1000}-\sum_{j=1}^{J} X_{(i 1) j}^{+} R E_{j}^{-} \leq O N_{(i 2)}^{+}-\frac{158.8 D_{(i 2) k}}{1000}-$

$\sum_{j=1}^{J} X_{(i 2) j}^{+} R E_{j}^{-}, i 1, i 2=1,2, \ldots, I ; k=1,2, \ldots, K$

$\sum_{j=1}^{J} X_{i j}^{+} \leq 1, i=1,2, \ldots, I$.

$X_{i j}^{+}=0$ or $1 ; i=1,2, \ldots, I$, and $j=1,2, \ldots, J$.

Models 8 and 9 above can be solved by the solution proposed by Fan et al. (2012).

\section{Results and Discussion}

Table 4 presents the solutions obtained from the IBP model for noise abatement under uncertainty. The results show that different measures would be applied to different noise sources to mitigate the noise effect on nearby residences. Moreover, for one factory, the preferred noise control measure may be different under different acceptable noise levels.

Three scenarios have been considered for this problem. Scenario 1 is the strictest, with the lowest acceptable noise levels, Scenario 2 is the middle range and Scenario 3 has the highest accepted noise levels. The type of scenario applicable to the problem would depend on the type of communities nearby - a residential community with retirement homes, for example, should have lower acceptable noise levels than a commercial area.

Scenario 1 corresponds to the strictest standard, where both Communities 1 and 2 are exposed to the lowest acceptable noise levels. In this scenario, option 6 (i.e. Shelter and Resilience) would be applied to control noise for factory 1 under the optimistic condition, which corresponds to the lower bound of the objective-function value, while option 7 
Table 4. Solutions from IBP Model under Different Scenarios

\begin{tabular}{|c|c|c|c|c|c|c|c|c|c|c|c|c|}
\hline$X_{i j}$ & $j=1$ & $\mathrm{j}=2$ & $j=3$ & $j=4$ & $j=5$ & $j=6$ & $j=7$ & $j=8$ & $j=9$ & $\mathrm{j}=10$ & $j=11$ & $\mathrm{TC}$ \\
\hline \multicolumn{13}{|c|}{ Scenario 1} \\
\hline $\mathrm{i}=1$ & 0 & 0 & 0 & 0 & 0 & {$[1,0]$} & {$[0,1]$} & 0 & 0 & 0 & 0 & \multirow[t]{3}{*}[1100,1435]{} \\
\hline $\mathrm{i}=2$ & 0 & 0 & 0 & 0 & 0 & 0 & {$[1,0]$} & 0 & 0 & {$[0,1]$} & 0 & \\
\hline$i=3$ & 0 & 0 & 0 & 0 & {$[0,1]$} & 0 & 0 & {$[1,0]$} & 0 & 0 & 0 & \\
\hline \multicolumn{13}{|c|}{ Scenario 2} \\
\hline$i=1$ & 0 & 0 & 0 & {$[1,0]$} & 0 & {$[0,1]$} & 0 & 0 & 0 & 0 & 0 & \multirow[t]{3}{*}[900,1365]{} \\
\hline $\mathrm{i}=2$ & 0 & 0 & 0 & 0 & 0 & {$[1,0]$} & 0 & 0 & 0 & {$[0,1]$} & 0 & \\
\hline$i=3$ & 0 & 0 & 0 & 0 & {$[0,1]$} & 0 & 0 & 0 & 0 & {$[1,0]$} & 0 & \\
\hline \multicolumn{13}{|c|}{ Scenario 3} \\
\hline$i=1$ & 0 & 0 & 0 & 0 & 0 & {$[0,1]$} & 0 & 0 & {$[1,0]$} & 0 & 0 & \multirow[t]{3}{*}[860,1180]{} \\
\hline $\mathrm{i}=2$ & 0 & 0 & 0 & 0 & 0 & {$[1,0]$} & {$[0,1]$} & 0 & 0 & 0 & 0 & \\
\hline$i=3$ & 0 & 0 & 0 & 0 & 0 & 0 & 0 & {$[0,1]$} & 0 & {$[1,0]$} & 0 & \\
\hline
\end{tabular}

Table 5. Results for Scenario 1

\begin{tabular}{lllll}
\hline & Optimistic & \multicolumn{2}{l}{ Conservative } \\
\hline & Option & Cost & Option & Cost \\
\hline Source 1 & 6 & 260 & 7 & 350 \\
Source 2 & 7 & 320 & 10 & 435 \\
Source 3 & 8 & 520 & 5 & 650 \\
System Cost & 1100 & & 1435 & \\
\hline
\end{tabular}

Table 6. Results for Scenario 2

\begin{tabular}{lllll}
\hline & Optimistic & \multicolumn{2}{l}{ Conservative } \\
\hline & Option & Cost & Option & Cost \\
\hline Source 1 & 4 & 240 & 6 & 280 \\
Source 2 & 6 & 260 & 10 & 435 \\
Source 3 & 10 & 400 & 5 & 650 \\
System Cost & 900 & & 1365 & \\
\hline
\end{tabular}

Table 7. Results for Scenario 3

\begin{tabular}{lllll}
\hline & Optimistic & \multicolumn{2}{l}{ Conservative } \\
\hline & Option & Cost & Option & Cost \\
\hline Source 1 & 9 & 200 & 6 & 280 \\
Source 2 & 6 & 260 & 7 & 350 \\
Source 3 & 10 & 400 & 8 & 550 \\
System Cost & 860 & & 1180 & \\
\hline
\end{tabular}

would be considered under the conservative condition, as shown in Table 5.

This is because the optimistic condition would predominately focus on reducing system cost while the conservative condition mainly considers the effects of noise reduction. Under the optimistic condition, the noise-reduction effect is considered to achieve its upper bound (e.g. $14.5 \mathrm{~dB}$ for option 6) and the cost of each option goes to its lower bound (e.g. \$260 for option 6), as presented in Table 1. Consequently, the noise from factory 1 (i.e. noise source 1) can just be reduced through option 6 to satisfy the acceptable noise levels for these two communities.

Conversely, the conservative condition primarily wants to guarantee the noise control effect, which regards all control measures as achieving their lower bounds (e.g. $15 \mathrm{~dB}$ for option 7) and the cost of each control measure as achieving its upper bound (e.g. $\$ 350$ for option 7), as shown in Table 1 . Therefore, option 7 should be applied to control noise from source 1 to satisfy the standards of the two communities. For noise source 2, the noise control options would be similar to noise source 1. A less expensive option (i.e. option 7) is to be applied under the optimistic conditions while a more expensive option (option 10) is to be used under conservative conditions. For noise source 3, option 8 (i.e. Shelter and Barrier), which will cost less and has a lower efficiency, would be used to control noise under optimistic conditions while option 5 (i.e. Equipment Update), which is more expensive but more effective, would be applied under conservative conditions.

Scenario 2 would allow normal standards for both communities, indicating acceptable noise levels to be 61 and 56 $\mathrm{dB}$ for Communities 1 and 2, respectively (as shown in Table 2). In this scenario, the solution of $X_{14}^{ \pm}=[1,0]$ means that option 4 (i.e. Barrier) is used to control the noise from factory 1 (i.e. noise source 1) under optimistic conditions, as shown in Table 6.

The solution of $X_{16}^{ \pm}=[0,1]$ indicates that option 6 is applied to control the noise from source 1 under conservative conditions. Compared with the options for source 1 in scena-

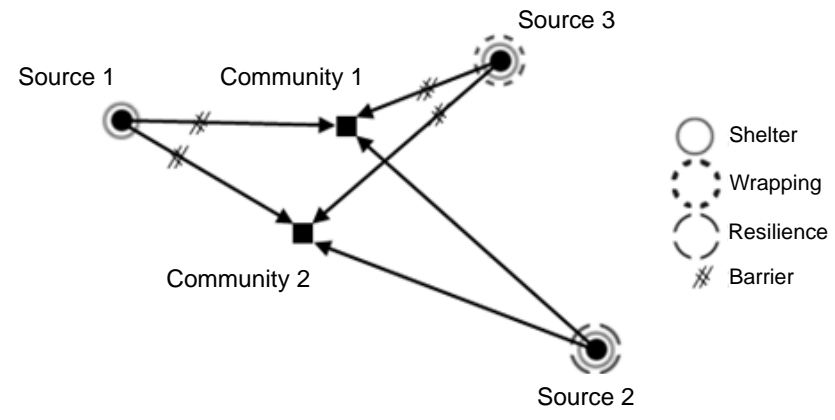

Figure 3. Noise control measures under Scenario 2. 


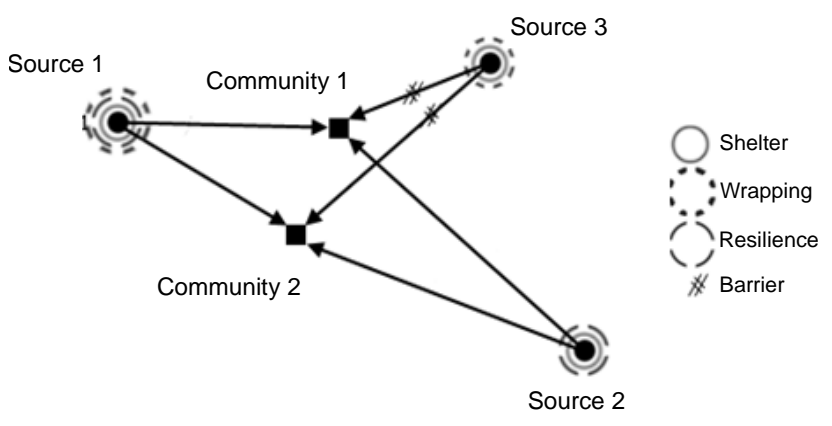

Figure 4. Noise control measures under Scenario 3.

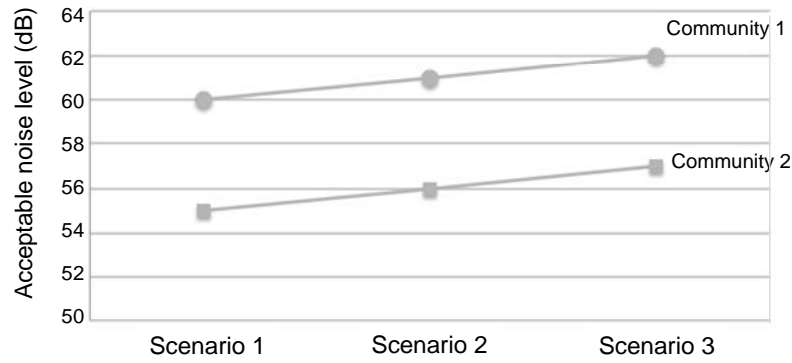

Figure 5. Acceptable noise levels for Communities 1 and 2.

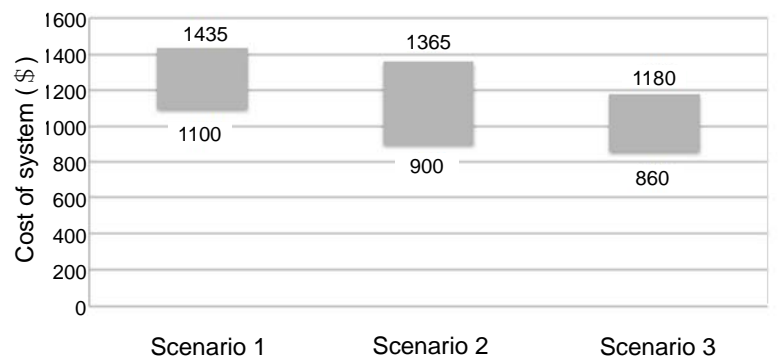

Figure 6. Upper and lower bounds of costs for recommended noise reduction systems.

rio 1 , the control measures in scenario 2 would be less effecttive but also less expensive due to the loosening of the acceptable noise level constraints for the two communities. For noise source 2, option $6\left(X_{26}^{ \pm}=[1,0]\right)$ would be applied to control its noise under less stringent conditions, while the same option as scenario 1 (i.e. option 10) would be used under more stringent noise control requirements. For noise source 3, the main difference for control measures between scenarios 1 and 2 is that a less effective option $\left(X_{310}^{ \pm}=[1,0]\right)$ is desirable under advantageous conditions. Under demanding conditions, the noise control measure would be the same (i.e. option 5) for these two scenarios.

In scenario 3, which implements the loosest noise control standards in two communities, the main difference for noise control measures for three sources under optimistic conditions is that source 1 requires a less effective and less expensive measure (i.e. $X_{19}^{ \pm}=[1,0]$ with a cost of $\$[200,220]$ and a noise-reduction effect of $[10,11] \mathrm{dB})$. Under conservative conditions, both sources 2 and 3 would change to some less effective noise control measures due to the relaxation of the standards. Options 7 and 8 (i.e. $X_{27}^{ \pm}=[0,1]$ and $X_{38}^{ \pm}=[0,1]$ ) would be applied to noise sources 2 and 3, respectively.

Generally, the above results indicate that through the proposed modeling approach, uncertainties presented as intervals in parameters can be communicated into the IBP model process. Table 4 provides the total system cost from the IBP model under different scenarios. The results suggest that different acceptable noise levels for the two communities would lead to varied objective function values. The system cost would have an opposite tendency to that of the acceptable noise levels for the two communities. As shown in Figures 5 and 6, both the lower and upper bounds of the system cost will decrease as acceptable noise levels increase. This is because as higher acceptable noise levels are implemented, lower effective noise control measures are required, and thus system costs are lowered.

Under each scenario, the lower-bound cost corresponds to optimistic conditions where noise control measures are assumed to be most effective, while the upper-bound cost is associated with more demanding conditions where the same measures are assumed to be less effective. For example, the system cost would be $\$[1,100,1,435]$ under scenario 1 , indicating that the system cost would be $\$ 1100$ under advantageous conditions and $\$ 1,435$ under demanding conditions. Moreover, the system cost would fluctuate within $\$ 1,100$ and $\$ 1,435$ as the model parameters vary within their lower and upper bounds. The system costs under the other two scenarios would have similar characteristics as that under scenario 1. Furthermore, the lower bound of the system cost is obtained under such consideration that each noise control measure would achieve the upper bound for the noise-reduction effect. Therefore, this may generate the highest risk of violating the acceptable noise levels of two communities. Conversely, the upper bound of the system cost is obtained with the most conservative noise-reduction effect of each control measure to be considered. This would definitely guarantee acceptable noise levels to be satisfied but may lead to excessive costs. Therefore, decision makers can make tradeoffs between system costs and the violation risk of acceptable noise levels, based on the solutions from the IBP model.

\section{Conclusions}

An interval binary programming (IBP) method has been proposed and applied to a representative noise control problem. As an extension of the binary programming method, IBP can explicitly address complexities and uncertainties in a noise control system. Parameters in the IBP model can be expressed as intervals, and also such uncertainties can be effectively incorporated within the model solution process. Two submodels corresponding to the lower and upper bounds of the objective-function value would be obtained based on an interactive algorithm, and interval solutions are then generated by solving the two submodels sequentially.

Results of the model application indicate that useful solutions for noise control practices can be generated. A num- 
ber of decision alternatives have been obtained and analyzed under different acceptable noise levels for the two communities. They reflect complex tradeoffs between environmental and economic considerations. A willingness to pay higher operating costs will guarantee meeting the noise control standards; however, a desire to reduce the costs will run into the risk of potentially violating acceptable noise levels.

Although this study is a new application for the IBP methodology, the results suggest that this approach is applicable to practical noise control problems that are associated with highly complex and uncertain information.

\section{Appendix 1}

Definition 1. Let $x$ denote a closed and bounded set of real number. An interval number $x^{ \pm}$is defined as an interval with known lower and upper bounds but unknown distribution information for $x$ (Huang et al., 1992):

$x^{ \pm}=\left[x^{-}, x^{+}\right]=\left\{t \in x \mid x^{-} \leq t \leq x^{+}\right\}$

where $x^{-}$and $x^{+}$are the lower and upper bounds of $x^{ \pm}$, respectively. When $x^{-}=x^{+}, x^{ \pm}$becomes a deterministic number, i.e. $x^{ \pm}=x^{-}=x^{+}$.

Definition 2. Let $R$ ' denote a set of real integer numbers. An interval integer is an interval number with integer lower and upper bounds, and all of its elements are integers (Huang et al., 1995):

$y^{ \pm}=\left[y^{-}, y^{+}\right], y^{-} \in R^{\prime}, y^{+} \in R^{\prime}$ any y $\in y^{ \pm}, y \in R^{\prime}$

Definition 3. An interval binary number is an interval integer with its two bounds being 0 and 1 , and its elements can only be 0 or 1 (Huang et al., 1992).

Definition 4. Let $* \in\{+,-, \times, \div\}$ be a binary operation on interval numbers. For interval numbers $x^{ \pm}$and $y^{ \pm}$, we have (Huang et al., 1995):

$x^{ \pm *} y^{ \pm}=\left[\min \left(x^{*} y\right), \max \left(x^{*} y\right)\right], x^{-} \leq x \leq x^{+}, y^{-} \leq y \leq y^{+}$

In the case of division, it is assumed that $\forall y \neq 0$. Hence we have:

$$
\begin{aligned}
& x^{ \pm}+y^{ \pm}=\left[x^{-}+y^{-}, x^{+}+y^{+}\right] \\
& x^{ \pm}-y^{ \pm}=\left[x^{-}-y^{+}, x^{+}-y^{-}\right] \\
& x^{ \pm} \times y^{ \pm}=[\min (x \times y), \max (x \times y)], x^{-} \leq x \leq x^{+}, y^{-} \leq y \leq \\
& y^{+} \\
& x^{ \pm} \div y^{ \pm}=[\min (x \div y), \max (x \div y)], x^{-} \leq x \leq x^{+}, y^{-} \leq y \leq \\
& y^{+}
\end{aligned}
$$

Definition 5. For an interval number, we have (Huang et al., 1995): $x^{ \pm} \leq y^{ \pm}$iff $x^{-} \leq y^{-}$and $x^{+} \leq y^{+}$

$x^{ \pm}<y^{ \pm}$iff $x^{-}<y^{-}$and $x^{+}<y^{+}$

Definition 6. For $x^{ \pm}=\left[x^{-}, x^{+}\right]$and $y^{ \pm}=\left[y^{-}, y^{+}\right]$, we have their order relations as follows (Huang et al., 1995):

$x^{ \pm} \leq y^{ \pm}$iff $x^{-} \leq y^{-}$and $x^{+} \leq y^{+}$

$x^{ \pm}<y^{ \pm}$iff $x^{-}<y^{-}$and $x^{+}<y^{+}$,

Definition 7. For an interval number $x^{ \pm}$, we define $\operatorname{Sign}\left(x^{ \pm}\right)$as follows (Huang et al., 1995):

$\operatorname{Sign}\left(\mathrm{x}^{ \pm}\right)=\left\{\begin{array}{l}1 \quad \text { if } \mathrm{x}^{ \pm} \geq 0 \\ -1 \text { if } \mathrm{x}^{ \pm}<0\end{array}\right.$

Definition 8. For an interval number $x^{ \pm}$, we define its grey absolute value $|x|^{ \pm}$as follows (Huang et al., 1995):

$|x|^{ \pm}= \begin{cases}|x|^{ \pm} & \text {if } x^{ \pm} \geq 0 \\ -|x|^{ \pm} & \text {if } x^{ \pm}<0\end{cases}$

Thus we have

$|\mathrm{x}|^{-}= \begin{cases}|\mathrm{x}|^{-} & \text {if } \mathrm{x}^{ \pm} \geq 0 \\ -|\mathrm{x}|^{+} & \text {if } \mathrm{x}^{ \pm}<0\end{cases}$

and

$|\mathrm{x}|^{+}= \begin{cases}|\mathrm{x}|^{+} & \text {if } \mathrm{x}^{ \pm} \geq 0 \\ -|\mathrm{x}|^{-} & \text {if } \mathrm{x}^{ \pm}<0\end{cases}$

\section{References}

Achterberg, T. (2007). Conflict analysis in mixed integer programming. Discrete Optimiz., 4(1), 1-20. http://dx.doi.org/10.1016/j.dis opt.2006.10.006

Asawarungsaengkul, K., and Nanthavanij, S. (2006). Design of Optimal Noise Hazard Control Strategy with Budget Constraint. Int. J. Occup. Saf. Ergonomics, 12(4), 355-367. PMid:17156611

Asawarungsaengkul, K., and Nanthavanij, S. (2007). A Genetic Algorithm Approach to the Selection of Engineering Controls for Optimal Noise Redution. ScienceAsia, 33, 89-101 http://dx.doi.org/ 10.2306/scienceasia1513-1874.2007.33.089

Baetz, B.W. (1990). Optimization/simulation modelling for waste management capacity planning. J. Urban Plann. Dev., 116(2), 5979. http://dx.doi.org/10.1061/(ASCE)0733-9488(1990)116:2(59)

Blair, C.E., and Jeroslow, R.G. (1982). The value function of an integer program. Math. Program., 23, 237-273. http://dx.doi.org/10.10 07/BF01583794

Chen, Z.L., Li, S.L., and Tirupati, D. (2002). A scenario-based stochastic programming approach for technology and capacity planning. Comput. Oper. Res., 29(7), 781-806. http://dx.doi.org/10. 1016/S0305-0548(00)00076-9 
Fan, Y.R., and Huang, G.H. (2012). A robust two-step method for solving linear programming problems within an environmental management context. J. Environ. Inf., 19(1), 1-9. http://dx.doi.org/ 10.3808/jei. 201200203

Garfinkel, R.S., and Nemhauser, G.L. (1972). Integer programming. John Wiley and Sons, New York.

Huang, G.H., Baetz, B.W., and Patry, G.G. (1992). A grey linear programming approach for municipal solid waste management planning under uncertainty. Civ. Eng. Syst., 9(4), 319-335. http://dx. doi.org/10.1080/02630259308970119

Huang, G.H., Baetz, B.W., and Patry, G.G. (1995). Grey integer programming: An application to waste management planning under uncertainty. Eur. J. Oper. Res., 83(3), 594-620. http://dx.doi. org/10.1016/0377-2217(94)00093-R

Jenkins, L. (1982). Parametric Mixed-Integer Programming: An Application to Solid Waste Management. Manage. Sci., 28(11), 12701284. http://dx.doi.org/10.1287/mnsc.28.11.1270

King, R.P., and Davis, J.R. (2003). Community noise: Health effects and management. Int. J. Hyg. Environ. Health, 206(2), 123-131. http://dx.doi.org/10.1078/1438-4639-00202

Mun, S., and Cho, Y.H. (2009). Noise barrier optimization using a simulated annealing algorithm. Appl. Acoust., 70(8), 1094-1098. http://dx.doi.org/10.1016/j.apacoust.2009.02.004

Murray-Johnson, L., Witte, K., Patel, D., Orrego, V., Zuckerman, C., Maxfield, A.M., and Thimons, E.D. (2004). Using the Extended Parallel Process Model to prevent noise-induced hearing loss among coal miners in Appalachia. Health Educ. Behav., 31(6), 741-55. http://dx.doi.org/10.1177/1090198104263396 National Institute for Occupational Safety and Health (1998). Health Hazard Evaluations: Noise and Hearing Loss, 1986-1997. DHHS Publication NO. 98-126, Cincinnati, Ohio.

Prats, X., Vicenc, P., Quevedo, J. (2011). A multi-objective optimization strategy for designing aircraft nois abatement procedures. Case study at Girona airport. Transp. Res. D: Transp. Environ., 16(1), 31-41. http://dx.doi.org/10.1016/j.trd.2010.07.007

Rajagopalan, S., Singh, M., and Morton, T.E. (1998). Capacity Expansion and Replacement with Uncertain Technological Breakthroughs. Manage. Sci., 44(1), 12-30 http://dx.doi.org/10.1287/mn sc. 44.1 .12

Tokmechi, Z. (2011). Uses of Monte Carlo Method for Noise Pollution Optimization. Adv. Environ. Biol., 5(9), 2937-2943.

Waly, S.M., and Sarker, B.R. (1998). Noise Reduction Using Nonlinear Optimization Modeling. Comput. Ind. Eng., 35(1), 327-330. http://dx.doi.org/10.1016/S0360-8352(98)00086-2

Yeh, L.J., Chang, Y.C., and Chiu, M.C. (2004). Optimization of allocation and noise redution on multi-system by using genetic algorithm. Noise Vibration Worldwide, 35(4), 11-18. http://dx.doi. org/10.1260/0957456041271243

Zachary, D.S., Gervais, J., and Leopold, U. (2010). Multi-impact optimization to reduce aviation noise and emissions. Transp. Res. D: Transp. Environ., 15(2), 82-93. http://dx.doi.org/ 10.1016/j.trd. 2009.09.005 